\title{
Evaluating the Performance of Various ACOPF Formulations Using Nonlinear Interior-Point Method
}

This paper was downloaded from TechRxiv (https://www.techrxiv.org).

LICENSE

CC BY 4.0

SUBMISSION DATE / POSTED DATE

29-07-2021 / 02-08-2021

CITATION

Sadat, Sayed Abdullah (2021): Evaluating the Performance of Various ACOPF Formulations Using Nonlinear Interior-Point Method. TechRxiv. Preprint. https://doi.org/10.36227/techrxiv.15078552.v1

$\mathrm{DOI}$

10.36227/techrxiv.15078552.v1 


\title{
Evaluating the Performance of Various ACOPF Formulations Using Nonlinear Interior-Point Method
}

\author{
Sayed Abdullah Sadat \\ Department of Electrical and Computer Engineering \\ University of Utah \\ email:sayed_abdullah@ieee.org
}

\begin{abstract}
Alternating current optimal power flow (ACOPF) problem is a non-convex and a nonlinear optimization problem. Similar to most nonlinear optimization problems, ACOPF is an NP-hard problem. On the other hand, Utilities and independent service operators (ISO) require the problem to be solved in almost real-time. The real-world networks are often large in size and developing an efficient and tractable algorithm is critical to many decision-making processes in electricity markets. Interiorpoint methods (IPMs) for nonlinear programming are considered one of the most powerful algorithms for solving large-scale nonlinear optimization problems. However, the performance of these algorithms is significantly impacted by the optimization structure of the problem. Thus, the choice of the formulation is as important as choosing the algorithm for solving an ACOPF problem. Different ACOPF formulations are evaluated in this paper for computational viability and best performance using the interior-point line search (IPLS) algorithm. Different optimization structures are used in these formulations to model the ACOPF problem representing a range of varying sparsity. The numerical experiments suggest that the least sparse ACOPF formulation with polar voltages yields the best computational results. A wide range of test cases, ranging from 500-bus systems to 9591-bus systems, are used to verify the test results.
\end{abstract}

Index Terms-ACOPF, interior-point methods, optimal power flow, line search algorithm, sparsity, power system economics, ACOPF formulations.

\section{INTRODUCTION}

$\mathbf{T}$ HE aim of power system operation is to deliver power to customers in a reliable and cost-effective manner. Optimal and reliable power dispatch is an optimization problem, which in its original form is referred to as the "alternating current optimal power flow" (ACOPF) problem [1], [2]. ACOPF is a nonlinear optimization problem with non-convex constraints, which makes it NP-hard [3].

The computational challenge is also significant because of the large size of real-world networks. The power grid in the United States is the largest machine ever built by humans, with hundreds of thousands of buses, transmission elements, and generators in three synchronous interconnections. According to the U.S. Energy Information Administration, the aggregate sum of annual retail transactions in the US power grid now exceeds USD 400 billion [4]. Clearly, a cost-effective operation is important due to the economic impacts of this crucial infrastructure.

Because of the difficulty of solving the ACOPF problem, the majority of the grid operators use some form of a direct current optimal power flow (DCOPF) formulation [5]. The
DCOPF linearization used in electricity market problems is based on a few assumptions that help convert the nonlinear ACOPF problem to a linear formulation [6], [7]. Although the implementations enable solving optimal power flow within the practical time constraints, rough approximations of voltage and reactive power flow are applied, requiring the application of conservative transmission limits. This can most likely result in an inefficient and sub-optimal operation of the grid.

According to a staff report by the U.S. Federal Energy Regulatory Commission (FERC), the differences between DCOPF and more efficient operations that may be achieved with ACOPF may incur $10 \%$ additional cost due to the suboptimal operation of the grid [1]. In the United States, the economic costs represent tens of billions of dollars each year. Moreover, a good solution to ACOPF is critical to many complex decision-making processes, including consideration of trade-offs between active and reactive power flows [8], [9]. Thus, developing a method that efficiently solves the ACOPF problem can have a significant economic impact.

Over the past few decades, many methods have been proposed to solve the ACOPF problems. However, the majority of them overlook the impact of formulation on the performance of ACOPF solutions, especially when solving large-scale problems. Many methods have been proposed and tested for solving ACOPF, including a variety of convex relaxation techniques [10]-[13]. Another approach that has recently attracted a growing interest among researchers and power engineers is nonlinear programming (NLP) methods [14]-[22].

Finally, in [23] different optimal power flow formulations are presented and [24] numerically analyzed the performance of three different ACOPF formulations. However, the analysis are limited to branch flow models. These models do not encompass the least sparse formulations for an ACOPF problem.

The past work presents a great deal of progress in solving ACOPF problems by presenting different choices for the selection of an efficient algorithm. However, the performance of these algorithms can be significantly enhanced by the appropriate choice of the ACOPF formulation. None of the past work extensively explores the impact of formulation structure on the performance of their proposed algorithm, the impact of which could be significant, especially for large-scale problems.

This paper explicitly evaluates the performance of seven 
different ACOPF formulations with different optimization structures. The study includes both branch flow and nodal injection models for an ACOPF problem. The performance of these formulations are evaluated using the Interior-Point Optimizer (IPOPT) implementation of interior-point nonlinear programming method. According to [25], IPLS is a powerful algorithm for solving large-scale nonlinear optimization problems. IPOPT efficiently implements the IPLS filter method, which is the state-of-the-art method, and it is typically used as a benchmark by most of the recent work on ACOPF algorithms [3], [26], [27]. The contribution of this paper can be summarized as follows:

1) Formulation of ACOPF problem with different optimization structures ranging from most sparse to least sparse formulations.

2) Evaluation of the performance of different ACOPF formulations and the complexity of their sub-problems.

3) Finally, identification of the computationally best performing formulations.

Similarly, different techniques, equipment, solvers, and algorithms can have different performance behavior corresponding to different ACOPF formulations and an overall computational impact on ACOPF solution. Some of the techniques include employing box-constraints, different linear solvers, and active-set. Furthermore, shunt FACTS devices can also have a computational impact on ACOPF by availing additional reactive power at different nodes. Finally, The convergence pattern might differ when used in other methods. Thus, as a future work it is worth investigating the performance of different ACOPF formulations using different techniques, equipment, solvers and algorithms such as sequential linear programming (SLP), sequential quadratic programming (SQP), and alternating direction method of multipliers (ADMM).

The rest of the paper is organized as follows. In Section II, optimal power flow and its different formulations are introduced. In section III, the results of numerical experiments are presented. Finally, in section IV the paper is concluded.

\section{Optimal POWER Flow (OPF)}

An optimal power flow constitutes the core of power system operations. It is an optimization extension of the power flow analysis [28], where in addition to feasibility, an optimal dispatch is also desired. The objective of power system operation is to serve the customers at the minimum cost, taking into consideration the physical constraints of the system. This can be expressed as an optimization problem, referred to as optimal power flow (OPF). In addition to power system operations, a solution to the optimal power flow is also essential for power system planning. In an ACOPF problem, the most important equations are about the representation of transmission branches. There are different approaches to model a transmission branch in a power system. The most commonly used representation of the transmission branch is called the $\pi$-model, which is applied in this paper.

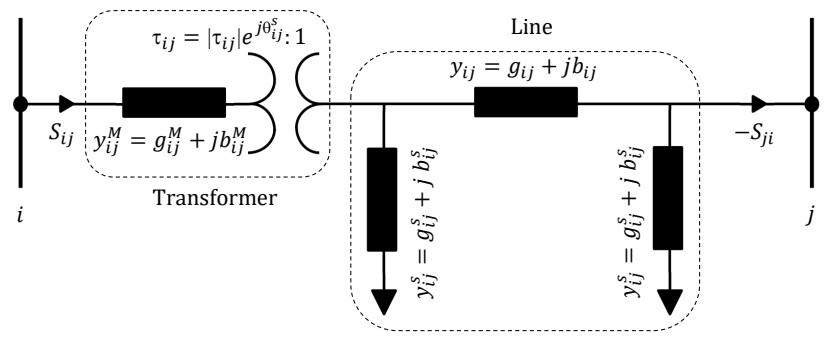

Fig. 1: A general representation of a $\pi$-model of a transmission branch, where $y_{i j}$ is the branch series admittance, $y_{i j}^{s}$ is the branch shunt admittance, $y_{i j}^{M}$ is the branch mutual admittance, and $\tau_{i j}$ is the branch complex tap ratio. If a branch is only a transmission line then $\tau_{i j}^{i}=1$ and $y_{i j}^{M}=0$. If a branch is only a transformer, then all the line parameters are zero. Thus, the figure represents a general model that can represent any type of transmission branch by simply adjusting the values of the parameters shown in the figure.

Let $y_{i j}^{n e t}=y_{i j}^{M}+\left(y_{i j}+y_{i j}^{s}\right) \cdot\left|\tau_{i j}^{i}\right|^{2}, y_{j i}^{n e t}=y_{i j}+y_{i j}^{s}$, and $2 \mathcal{K}=\{i, j\} \in \mathcal{K} \bigcup\{j, i\} \in \mathcal{K}$. The admittance matrix can be written as shown in (1).

$$
\begin{aligned}
Y_{i i} & =\sum_{\substack{k=1 \\
k \neq i}}^{\mathcal{K}_{i}^{f}} y_{i k}^{n e t}+\sum_{\substack{k=1 \\
k \neq i}}^{\mathcal{K}_{i}^{t}} y_{k i}^{n e t}, \\
Y_{i j} & =-y_{i j} \cdot \tau_{i j}^{i *} \\
Y_{j i} & =-y_{i j} \cdot \tau_{i j}^{i}
\end{aligned}
$$

An ACOPF problem can be formulated as an optimization problem as shown in (2).

$$
\begin{array}{ll}
\min & \sum_{g \in \mathcal{G}} c_{2 g} \cdot\left(\Re\left[s_{g}\right]\right)^{2}+c_{1 g} \cdot \Re\left[s_{g}\right]+c_{0 g} \\
\text { s.t. } & s_{i j}=v_{i} \cdot\left(y_{i j}^{n e t} \cdot v_{i}+Y_{i j} \cdot v_{j}\right)^{*}, \forall\{i, j\} \in 2 \mathcal{K} . \\
& \sum_{g \in \mathcal{G}_{n}} s_{g}-\sum_{d \in \mathcal{D}_{n}} s_{d}=\sum_{k \in \mathcal{K}_{n}^{f}} s_{n k}+\sum_{k \in \mathcal{K}_{n}^{t}} s_{k n} \\
& \quad+Y_{n}^{L} \cdot\left|v_{n}\right|^{2}, \forall n \in \mathcal{N} . \\
& V_{n}^{\text {Min }} \leq\left|v_{n}\right| \leq V_{n}^{\text {Max }}, \forall n \in \mathcal{N} . \\
& \left|s_{i j}\right| \leq I_{i j}^{\text {Max }} \cdot\left|v_{n}\right|, \forall\{i, j\} \in 2 \mathcal{K} . \\
& s_{g}^{\text {Min }} \leq s_{g} \leq s_{g}^{\text {Max }}, \forall g \in \mathcal{G} .
\end{array}
$$

Equation (2) represents one of the general representations of an ACOPF problem in its complex form based on the grid model presented in [29]. $c_{2 g}, c_{1 g}$, and $c_{0 g}$ are the coefficients of quadratic cost function of generator $g, s_{g}=p_{g}+j q_{g}$ is the apparent power output of generator $g, v_{n}=v_{n}^{r}+j v_{n}^{i}=$ $V_{n} e^{j \theta_{n}}$ is the voltage at bus $n, S_{i j}=P_{i j}+j Q_{i j}$ is the line apparent power flow from node $i$ to $j, s_{d}=p_{d}+j q_{d}$ is the complex load, $V_{n}^{\text {Min }}$ and $V_{n}^{\text {Max }}$ are lower and upper voltage bounds at bus $n, I_{i j}^{M a x}$ is the current thermal limit of line $\{i, j\}, s_{g}^{\text {Min }}=p_{g}^{M i n}+j q_{g}^{\text {Min }}, s_{g}^{\text {Max }}=p_{g}^{\text {Max }}+j q_{g}^{\text {Max }}$ are the complex generator $g$ bounds, and $Y_{n}^{L}=G_{n}^{L}-j B_{n}^{L}$ is the complex Shunt element connected to bus $n . \mathcal{N}$ is the set of 
buses, $\mathcal{G}$ is the set of generators, and $\mathcal{K}$ is the set of branches in the network.

Power generation output can be separated into real and reactive power components and therefore, the constraints in (2f) can be written as (3).

$$
\begin{aligned}
& p_{g}^{\text {Min }} \leq p_{g} \leq p_{g}^{\text {Max }}, \forall g \in \mathcal{G} \\
& q_{g}^{\text {Min }} \leq q_{g} \leq q_{g}^{\text {Max }}, \forall g \in \mathcal{G} .
\end{aligned}
$$

An ACOPF problem in its complex form can be expanded into different solvable optimization formulations as shown in fig. 2. While these formulations represent the same original ACOPF problem, they have different optimization structures that can have different convergence patterns. The ACOPF formulations can be broadly classified as either of the following.

1) Branch Flow Models

2) Nodal Injection Models

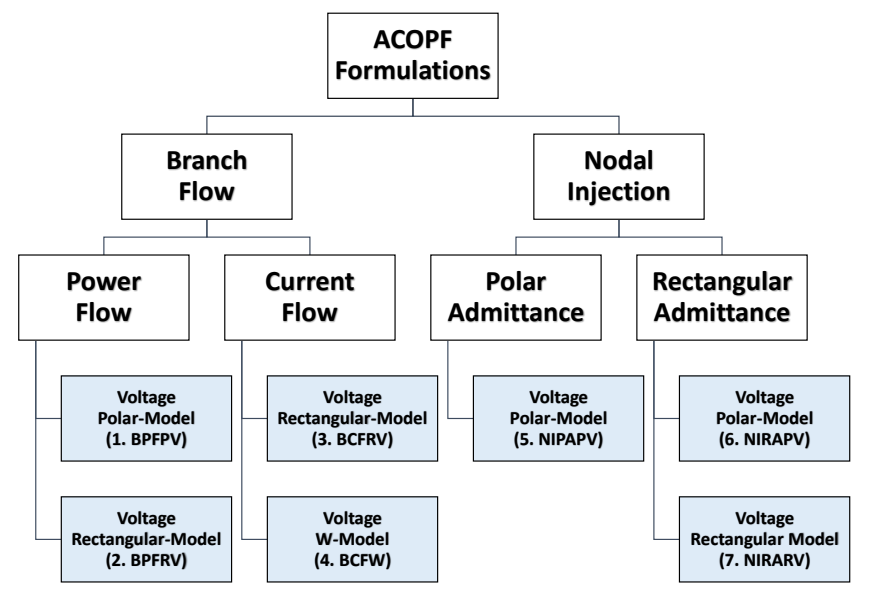

Fig. 2: Classification of different ACOPF formulations.

In addition, the flow can be either represented by power equations or current equations. Finally, voltages are either represented by polar form, rectangular form, or $W$-model in modeling different ACOPF formulations.

\section{A. Branch Flow Model}

In a branch flow model ACOPF problem, a power or current flow in each branch is explicitly represented as variables. To use the power equations, the branches can be modeled as (4).

$$
s_{i j}=v_{i} \cdot i_{i j}^{*}=v_{i} \cdot\left(y_{i j}^{n e t} \cdot v_{i}+Y_{i j} \cdot v_{j}\right)^{*}, \forall\{i, j\} \in 2 \mathcal{K}
$$
(5).

To use current equations, the branches can be modeled as

$$
i_{i j}=y_{i j}^{n e t} \cdot v_{i}+Y_{i j} \cdot v_{j}, \forall\{i, j\} \in 2 \mathcal{K}
$$

These branch variables are then used in the node balance equations.

\section{B. Nodal Injection Model}

In this model, flows are not represented by explicit variables and instead are integrated in node power balance equations using admittance matrix and voltages as shown in (6).

$$
\begin{aligned}
\sum_{g \in \mathcal{G}_{n}} s_{g}-\sum_{d \in \mathcal{D}_{n}} s_{d}= & v_{n} \cdot \sum_{k \in \mathcal{N}} Y_{n k}^{*} \cdot v_{k}^{*} \\
& +Y_{n}^{L} \cdot\left|v_{n}\right|^{2}, \forall n \in \mathcal{N} .
\end{aligned}
$$

Three different approaches for modeling voltages in an ACOPF problem are proposed.

of

\section{Voltage Polar Form}

In this category, the voltages are modeled in polar form. Using the polar form of the voltages in ACOPF problem, the branch power flow can be modeled as shown in (7).

$$
\begin{aligned}
P_{i j} & =g_{i j}^{n e t} \cdot V_{i}^{2}+\left(G_{i j} \cdot \cos \left(\theta_{i}-\theta_{j}\right)\right. \\
& +B_{i j} \cdot \sin \left(\theta_{i}-\theta_{j}\right) \cdot V_{i} \cdot V_{j}, \forall\{i, j\} \in 2 \mathcal{K} . \\
Q_{i j} & =-b_{i j}^{n e t} \cdot V_{i}^{2}+\left(G_{i j} \cdot \sin \left(\theta_{i}-\theta_{j}\right)\right. \\
& -B_{i j} \cdot \cos \left(\theta_{i}-\theta_{j}\right) \cdot V_{i} \cdot V_{j}, \forall\{i, j\} \in 2 \mathcal{K} .
\end{aligned}
$$

Correspondingly, the node power balance can be written as shown in (8).

$$
\begin{aligned}
\sum_{g \in \mathcal{G}_{n}} p_{g}-\sum_{d \in \mathcal{D}_{n}} p_{d} & =\sum_{k \in \mathcal{K}_{n}^{f}} P_{n k}+\sum_{k \in \mathcal{K}_{n}^{t}} P_{k n} \\
& +G_{n}^{L} \cdot V_{n}^{2}, \quad \forall n \in \mathcal{N} . \\
\sum_{g \in \mathcal{G}_{n}} q_{g}-\sum_{d \in \mathcal{D}_{n}} q_{d} & =\sum_{k \in \mathcal{K}_{n}^{f}} Q_{n k}+\sum_{k \in \mathcal{K}_{n}^{t}} Q_{k n} \\
& -B_{n}^{L} \cdot V_{n}^{2}, \quad \forall n \in \mathcal{N} .
\end{aligned}
$$

For branch flow, the power thermal limit of transmission branches can be enforced as shown in (9).

$$
P_{i j}^{2}+Q_{i j}^{2} \leq\left(I_{i j}^{M a x}\right)^{2} \cdot V_{n}^{2}, \forall\{i, j\} \in 2 \mathcal{K} .
$$

Similarly, for the nodal injection models with polar admittance, the nodal power balance can be written as shown in (10).

$$
\begin{aligned}
\sum_{g \in \mathcal{G}_{n}} p_{g}-\sum_{d \in \mathcal{D}_{n}} p_{d} & =\sum_{k \in \mathcal{N}}\left|Y_{n k}\right| \cdot V_{n} \cdot V_{k} \cdot \cos \left(\theta_{n}-\theta_{k}\right. \\
& \left.-\theta_{n k}^{Y}\right)+G_{n}^{L} \cdot V_{n}^{2}, \forall n \in \mathcal{N} \\
\sum_{g \in \mathcal{G}_{n}} q_{g}-\sum_{d \in \mathcal{D}_{n}} q_{d} & =\sum_{k \in \mathcal{N}}\left|Y_{n k}\right| \cdot V_{n} \cdot V_{k} \cdot \sin \left(\theta_{n}-\theta_{k}\right. \\
& \left.-\theta_{n k}^{Y}\right)-B_{n}^{L} \cdot V_{n}^{2}, \forall n \in \mathcal{N}
\end{aligned}
$$

Similarly, for the nodal injection model with rectangular admittance, the nodal power balance can be written as shown in (11). 


$$
\begin{aligned}
\sum_{g \in \mathcal{G}_{n}} p_{g}-\sum_{d \in \mathcal{D}_{n}} p_{d} & =\sum_{k \in \mathcal{N}} V_{n} \cdot V_{k} \cdot\left(G_{n k} \cdot \cos \left(\theta_{n}-\theta_{k}\right)\right. \\
& \left.+B_{n k} \cdot \sin \left(\theta_{n}-\theta_{k}\right)\right) \\
& +G_{n}^{L} \cdot V_{n}^{2}, \forall n \in \mathcal{N} . \\
\sum_{g \in \mathcal{G}_{n}} q_{g}-\sum_{d \in \mathcal{D}_{n}} q_{d} & =\sum_{k \in \mathcal{N}} V_{n} \cdot V_{k} \cdot\left(G_{n k} \cdot \sin \left(\theta_{n}-\theta_{k}\right)\right. \\
& \left.-B_{n k} \cdot \cos \left(\theta_{n}-\theta_{k}\right)\right) \\
& -B_{n}^{L} \cdot V_{n}^{2}, \forall n \in \mathcal{N} .
\end{aligned}
$$

For nodal injection models, the current thermal limit of transmission branches can be enforced as shown in (12).

$$
\begin{aligned}
& 2 \cdot\left|y_{i j}^{n e t}\right| \cdot\left|Y_{i j}\right| \cdot V_{i} \cdot V_{j} \cdot[ \\
& \left.\cos \left(\theta_{n}-\theta_{k}+\theta_{n k}^{y Y}\right)\right] \\
& +\left|y_{i j}^{n e t}\right|^{2} \cdot V_{i}^{2}+\left|Y_{i j}\right|^{2} \cdot V_{j}^{2} \leq\left(I_{i j}^{M a x}\right)^{2}, \forall\{i, j\} \in 2 \mathcal{K} .
\end{aligned}
$$

The voltage magnitude limits can be enforced using (13).

$$
V_{n}^{M i n} \leq V_{n} \leq V_{n}^{\text {Max }}, \forall n \in \mathcal{N} .
$$

\section{Voltage Rectangular Form}

In this category, voltages are modeled in rectangular form. Using the rectangular form of the voltage in the ACOPF problem, the branch power flow can be modeled as shown in (14).

$$
\begin{aligned}
P_{i j} & =g_{i j}^{n e t} \cdot\left[\left(v_{i}^{r}\right)^{2}+\left(v_{i}^{i}\right)^{2}\right]+G_{i j} \cdot v_{i}^{r} \cdot v_{j}^{r}+B_{i j} \cdot v_{i}^{i} \cdot v_{j}^{r} \\
& +G_{i j} \cdot v_{i}^{i} \cdot v_{j}^{i}-B_{i j} \cdot v_{i}^{r} \cdot v_{j}^{i}, \forall\{i, j\} \in 2 \mathcal{K} . \quad(14 \mathrm{a}) \\
Q_{i j} & =-b_{i j}^{n e t} \cdot\left[\left(v_{i}^{r}\right)^{2}+\left(v_{i}^{i}\right)^{2}\right]+G_{i j} \cdot v_{i}^{i} \cdot v_{j}^{r}-B_{i j} \cdot v_{i}^{r} \cdot v_{j}^{r} \\
& -G_{i j} \cdot v_{i}^{r} \cdot v_{j}^{i}-B_{i j} \cdot v_{i}^{i} \cdot v_{j}^{i}, \forall\{i, j\} \in 2 \mathcal{K} . \quad(14 \mathrm{~b})
\end{aligned}
$$

Correspondingly, the node power balance can be written as shown in (15).

$$
\begin{aligned}
\sum_{g \in \mathcal{G}_{n}} p_{g}-\sum_{d \in \mathcal{D}_{n}} p_{d} & =\sum_{k \in \mathcal{K}_{n}^{f}} P_{n k}+\sum_{k \in \mathcal{K}_{n}^{t}} P_{k n} \\
& +G_{n}^{L} \cdot\left[\left(v_{n}^{r}\right)^{2}+\left(v_{n}^{i}\right)^{2}\right], \forall n \in \mathcal{N} . \\
\sum_{g \in \mathcal{G}_{n}} q_{g}-\sum_{d \in \mathcal{D}_{n}} q_{d} & =\sum_{k \in \mathcal{K}_{n}^{f}} Q_{n k}+\sum_{k \in \mathcal{K}_{n}^{t}} Q_{k n} \\
& -B_{n}^{L} \cdot\left[\left(v_{n}^{r}\right)^{2}+\left(v_{n}^{i}\right)^{2}\right], \forall n \in \mathcal{N} .
\end{aligned}
$$

For branch power flow, the thermal limit of transmission branches can be enforced as shown in (16).

$$
P_{i j}^{2}+Q_{i j}^{2} \leq\left(I_{i j}^{\text {Max }}\right)^{2} \cdot\left[\left(v_{n}^{r}\right)^{2}+\left(v_{n}^{i}\right)^{2}\right], \forall\{i, j\} \in 2 \mathcal{K} \text {. }
$$

Using the rectangular form of the voltage in the ACOPF problem, the branch current flow can be modeled as shown in (17).

$$
\begin{aligned}
i_{i j}^{r} & =g_{i j}^{n e t} \cdot v_{i}^{r}-b_{i j}^{n e t} \cdot v_{i}^{i}+G_{i j} \cdot v_{j}^{r} \\
& -B_{i j} \cdot v_{j}^{i}, \forall\{i, j\} \in 2 \mathcal{K} . \\
i_{i j}^{i} & =g_{i j}^{n e t} \cdot v_{i}^{i}+b_{i j}^{n e t} \cdot v_{i}^{r}+G_{i j} \cdot v_{j}^{i} \\
& +B_{i j} \cdot v_{j}^{r}, \forall\{i, j\} \in 2 \mathcal{K} .
\end{aligned}
$$

Correspondingly, the node power balance can be written as shown in (18).

$$
\begin{aligned}
\sum_{g \in \mathcal{G}_{n}} p_{g}-\sum_{d \in \mathcal{D}_{n}} p_{d} & =\sum_{\{n, k\} \in \mathcal{K}_{n}^{f} \cup \mathcal{K}_{n}^{t}}\left(v_{n}^{r} \cdot i_{n k}^{r}+v_{n}^{i} \cdot i_{n k}^{i}\right) \\
& +G_{n}^{L} \cdot\left[\left(v_{n}^{r}\right)^{2}+\left(v_{n}^{i}\right)^{2}\right], \forall n \in \mathcal{N} . \\
\sum_{g \in \mathcal{G}_{n}} q_{g}-\sum_{d \in \mathcal{D}_{n}} q_{d} & =\sum_{\{n, k\} \in \mathcal{K}_{n}^{f} \cup \mathcal{K}_{n}^{t}}\left(v_{n}^{i} \cdot i_{n k}^{r}+v_{n}^{r} \cdot i_{n k}^{i}\right) \\
& -B_{n}^{L} \cdot\left[\left(v_{n}^{r}\right)^{2}+\left(v_{n}^{i}\right)^{2}\right], \forall n \in \mathcal{N} .
\end{aligned}
$$

where $i_{i j}=i_{i j}^{r}+j i_{i j}^{i}$ is the complex line current flow from node $i$ to $j$.

For branch current flow, the thermal limit of transmission branches can be enforced as shown in (19).

$$
\left(i_{i j}^{r}\right)^{2}+\left(i_{i j}^{i}\right)^{2} \leq\left(I_{i j}^{M a x}\right)^{2}, \forall\{i, j\} \in 2 \mathcal{K} .
$$

Similarly, for the nodal injection model with rectangular admittance, the nodal power balance can be written as shown in (20).

$$
\begin{aligned}
\sum_{g \in \mathcal{G}_{n}} p_{g}-\sum_{d \in \mathcal{D}_{n}} p_{d} & =\sum_{k \in \mathcal{N}}\left(G_{n k} \cdot v_{n}^{r} \cdot v_{k}^{r}+B_{n k} \cdot v_{n}^{i} \cdot v_{k}^{r}\right. \\
& \left.+G_{n k} \cdot v_{n}^{i} \cdot v_{k}^{i}-B_{n k} \cdot v_{n}^{r} \cdot v_{k}^{i}\right) \\
& +G_{n}^{L} \cdot\left[\left(v_{n}^{r}\right)^{2}+\left(v_{n}^{i}\right)^{2}\right], \forall n \in \mathcal{N} . \\
\sum_{g \in \mathcal{G}_{n}} q_{g}-\sum_{d \in \mathcal{D}_{n}} q_{d} & =\sum_{k \in \mathcal{N}}\left(G_{n k} \cdot v_{n}^{i} \cdot v_{k}^{r}-B_{n k} \cdot v_{n}^{r} \cdot v_{k}^{r}\right. \\
& \left.-G_{n k} \cdot v_{n}^{r} \cdot v_{k}^{i}-B_{n k} \cdot v_{n}^{i} \cdot v_{k}^{i}\right) \\
& -B_{n}^{L} \cdot\left[\left(v_{n}^{r}\right)^{2}+\left(v_{n}^{i}\right)^{2}\right], \forall n \in \mathcal{N} .
\end{aligned}
$$

For nodal injection models, the thermal limit of transmission branches can be enforced as shown in (21).

$$
\begin{aligned}
& \left(g_{i j}^{n e t} \cdot v_{i}^{r}-b_{i j}^{n e t} \cdot v_{i}^{i}\right. \\
& \left.+G_{i j} \cdot v_{j}^{r}-B_{i j} \cdot v_{j}^{i}\right)^{2} \\
& +\left(g_{i j}^{n e t} \cdot v_{i}^{i}+b_{i j}^{n e t} \cdot v_{i}^{r}\right. \\
& \left.+G_{i j} \cdot v_{j}^{i}+B_{i j} \cdot v_{j}^{r}\right)^{2} \leq\left(I_{i j}^{M a x}\right)^{2}, \forall\{i, j\} \in 2 \mathcal{K} .
\end{aligned}
$$

The voltage magnitude limits can be enforced using (22).

$$
\left(V_{n}^{\text {Min }}\right)^{2} \leq\left(v_{n}^{r}\right)^{2}+\left(v_{n}^{i}\right)^{2} \leq\left(V_{n}^{\text {Max }}\right)^{2}, \forall n \in \mathcal{N} .
$$

\section{E. Voltage W-Model}

In this category, the square of voltage magnitudes are replaced by a variable notated as $W^{d}$. This will make the optimization structure of the problem further sparse. In this model, $V_{n}^{2}$ variables or its equivalent expressions are substituted with $W_{n}^{d}$ variables.

The node power balance for the branch current flow can be modeled as shown in (23). 
TABLE I: The table analyzes optimization structure of different ACOPF formulations with objective function (2a). Typically, for a transmission system $\mathcal{K}>\mathcal{N}>\mathcal{G}$.

\begin{tabular}{|c|l|c|c|c|}
\hline \hline Sno. & \multicolumn{1}{|c|}{ ACOPF Formulations } & Constraints & $\begin{array}{c}\text { Num. of total } \\
\text { constraints }\end{array}$ & $\begin{array}{c}\text { Num. of NL } \\
\text { constraints }\end{array}$ \\
\hline 1 & Branch Power Flow Polar Voltage (BPFPV) & $(3),(7),(8),(9),(13)$ & $4 \mathcal{G}+4 \mathcal{N}+6 \mathcal{K}$ & $2 \mathcal{N}+6 \mathcal{K}$ \\
\hline 2 & Branch Power Flow Rectangular Voltage (BPFRV) & $(3),(14),(15),(16),(22)$ & $4 \mathcal{G}+4 \mathcal{N}+6 \mathcal{K}$ & $4 \mathcal{N}+6 \mathcal{K}$ \\
\hline 3 & Branch Current Flow Rectangular Voltage (BCFRV) & $(3),(17),(18),(19),(22)$ & $4 \mathcal{G}+4 \mathcal{N}+6 \mathcal{K}$ & $4 \mathcal{N}+2 \mathcal{K}$ \\
\hline 4 & Branch Current Flow W-Model (BCFW) & $(3),(17),(23),(19),(24 a),(24 b)$ & $4 \mathcal{G}+5 \mathcal{N}+10 \mathcal{K}$ & $3 \mathcal{N}+6 \mathcal{K}$ \\
\hline 5 & Nodal Injection Polar Admittance Polar Voltage (NIPAPV) & $(3),(10),(12),(13)$ & $4 \mathcal{G}+4 \mathcal{N}+2 \mathcal{K}$ & $2 \mathcal{N}+2 \mathcal{K}$ \\
\hline 6 & Nodal Injection Rectangular Admittance Polar Voltage (NIRAPV) & $(3),(11),(12),(13)$ & $4 \mathcal{G}+4 \mathcal{N}+2 \mathcal{K}$ & $2 \mathcal{N}+2 \mathcal{K}$ \\
\hline 7 & Nodal Injection Rectangular Admittance Rectangular Voltage (NIRARV) & $(3),(20),(21),(22)$ & $4 \mathcal{G}+4 \mathcal{N}+2 \mathcal{K}$ & $4 \mathcal{N}+2 \mathcal{K}$ \\
\hline \hline
\end{tabular}

$$
\begin{aligned}
\sum_{g \in \mathcal{G}_{n}} p_{g}-\sum_{d \in \mathcal{D}_{n}} p_{d} & =\sum_{\{n, k\} \in \mathcal{K}_{n}^{f} \cup \mathcal{K}_{n}^{t}}\left(v_{n}^{r} \cdot i_{n k}^{r}+v_{n}^{i} \cdot i_{n k}^{i}\right) \\
& +G_{n}^{L} \cdot W_{n}^{d}, \forall n \in \mathcal{N} . \\
\sum_{g \in \mathcal{G}_{n}} q_{g}-\sum_{d \in \mathcal{D}_{n}} q_{d} & =\sum_{\{n, k\} \in \mathcal{K}_{n}^{f} \cup \mathcal{K}_{n}^{t}}\left(v_{n}^{i} \cdot i_{n k}^{r}+v_{n}^{r} \cdot i_{n k}^{i}\right) \\
& -B_{n}^{L} \cdot W_{n}^{d}, \forall n \in \mathcal{N} .
\end{aligned}
$$

The W-Model and the limits can be formed using the voltage relationship shown in (24).

$$
\begin{aligned}
& \left(V_{n}^{\text {Min }}\right)^{2} \leq W_{n}^{d} \leq\left(V_{n}^{\text {Max }}\right)^{2}, \forall n \in \mathcal{N} . \\
& W_{n}^{d}=\left(v_{n}^{r}\right)^{2}+\left(v_{n}^{i}\right)^{2}, \forall n \in \mathcal{N} .
\end{aligned}
$$

Table I tabulates all seven ACOPF formulations using different combination of the equations derived above. All seven formulations use (2a) as their objective function.

\section{NUMERICAL EXPERIMENTS}

Various numerical experiments were performed on an 8-core Intel(R) i9-9980 HK CPU @2.40 GHz with 32 GB RAM system to test and validate the performance of different ACOPF formulations. The programming for numerical experiments are implemented in Julia v1.5.3 using IPOPT v3.13.2 and JuMP v0.21.5. in a Linux Ubuntu operating system.

The numerical experiments were performed on a set of synthetic networks varying from 500-bus systems to 9591bus systems. Table II presents the description of different test cases used in the numerical experiments. The test results are tabulated in Table III and IV. Table III shows the computation time for each test case using different ACOPF formulations. Similarly, table IV shows the number of iterations needed to solve each test case using different ACOPF formulations.

\section{Performance Profile}

To assess the performance of different ACOPF formulations solved with IPOPT solver, we plot the performance profile [30] using different scenarios of a wide range of test cases shown in Table II.

Let $\mathcal{F}$ be the set of ACOPF formulations. For each problem $p \in \mathcal{P}$ and formulation $f \in \mathcal{F}$, let $t_{p f}$ be the solution time for solving problem $p$ using formulation $f$. We define the performance ratio

$$
r_{p f}:=\frac{t_{p f}}{\min _{f \in \mathcal{F}} t_{p f}}
$$

that represents the performance of formulation $f$ as compared with the best performance by any formulation on problem $p$. Now we define the performance of formulation $f$ on any given problem as the probability for formulation $f$ that a performance ratio $r_{p f}$ is within a factor $\tau$ of the best possible ratio; that is,

$$
\rho_{f}(\tau):=\frac{1}{|\mathcal{P}|}\left|\left\{p \in \mathcal{P}: r_{p f} \leq \tau\right\}\right|
$$

Using (26) we plot the performance profile of different ACOPF formulations as shown in fig. 3 .

The performance profile shows that IPLS algorithm, in general, converges faster with dense (least sparse) ACOPF formulations. Similarly, it also converges faster when parameters, particularly, voltages are modeled using polar form. Therefore, the best performing formulations are the least sparse formulations with polar voltages. Modeling current flow in branches instead of power also helps branch flow ACOPF formulations with rectangular voltages. However, using voltages in polar form always outperforms other branch flow ACOPF formulations presented in this paper.

TABLE II: The table describes the size of different test networks used in the numerical experiments.

\begin{tabular}{|c|c|c|c|}
\hline \hline Networks & Buses & Gens & Branches \\
\hline \hline Network_01R-040 & 500 & 90 & 594 \\
\hline Network_03R-050 & 793 & 210 & 912 \\
\hline Network_05R-018 & 2000 & 384 & 3639 \\
\hline Network_07R-122 & 2312 & 617 & 3013 \\
\hline Network_81R-050 & 3288 & 379 & 4871 \\
\hline Network_84R-100 & 4601 & 410 & 7304 \\
\hline Network_09R-070 & 4918 & 1340 & 6727 \\
\hline Network_12R-030 & 9591 & 365 & 15915 \\
\hline \hline
\end{tabular}

Finally, for better visualization and breakdown of performance for each ACOPF formulation, a bar chart is plotted in fig. 4 to show the computation time, and in fig. 5 to show the number of iterations needed to solve different ACOPF formulations for Network_12R-030, which is a large-sclae network. As can be seen in fig. 4 and 5, different ACOPF formulations can have different complexity of sub-problems in IPLS Algorithm for the same ACOPF problem. For instance, in Network $\_12 R-030$ the BPFPV formulation has the highest computation time while solving the ACOPF problem in fewer iterations than BCFRV and NIPARV. This shows that the 


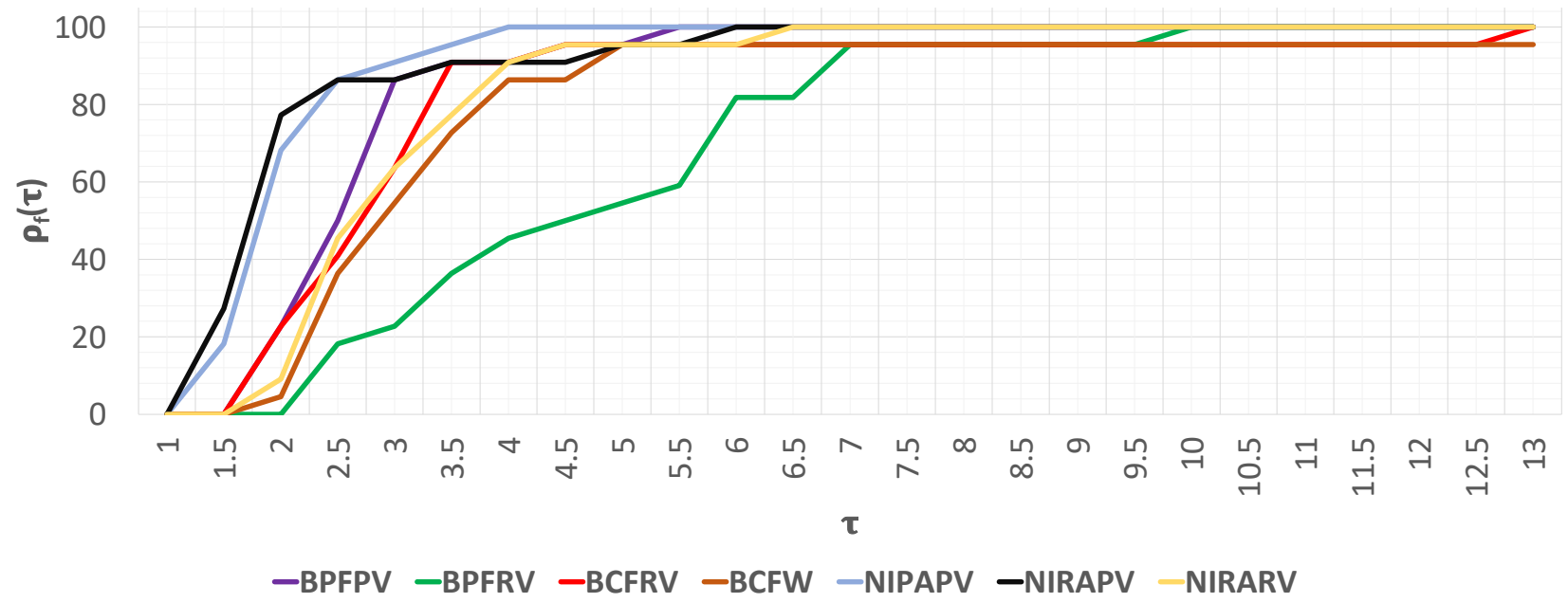

Fig. 3: Performance profile of different ACOPF formulations using IPOPT solver for different scenarios of a wide range of test cases shown in Table II.

TABLE III: The table presents the computation time of one scenario of various test networks using different ACOPF formulations.

\begin{tabular}{|c|c|c|c|c|c|c|c|}
\hline \hline Network & BPFPV & BPFRV & BCFRV & BCFW & NIPAPV & NIRAPV & NIPARV \\
\hline \hline Network_01R-040 & 3.73068 & 4.982912 & 4.076387 & 2.738256 & 1.91067 & 1.755969 & 3.081178 \\
\hline Network_03R-050 & 3.732486 & 3.773625 & 3.080637 & 3.440697 & 1.510793 & 1.443192 & 3.046712 \\
\hline Network_05R-018 & 16.363282 & 16.898092 & 412.73109 & 68.677693 & 14.070075 & 15.309972 & 28.015885 \\
\hline Network_07R-122 & 48.769313 & 59.856143 & 147.61875 & 67.495748 & 17.206433 & 15.67697 & 51.931805 \\
\hline Network_81R-050 & 96.409814 & 82.167673 & 66.547177 & 81.969888 & 36.648551 & 33.459432 & 57.04276 \\
\hline Network_84R-100 & 210.508382 & 175.200104 & 163.442526 & 152.807441 & 40.084922 & 43.241946 & 73.861782 \\
\hline Network_09R-070 & 44.880277 & 46.045293 & 39.612629 & 47.888543 & 22.53276 & 19.634313 & 38.124695 \\
\hline Network_12R-030 & 561.562603 & 368.828423 & 457.325533 & 392.646498 & 122.726361 & 133.988779 & 484.546118 \\
\hline \hline
\end{tabular}

TABLE IV: The table presents the number of barrier iterations needed to solve one scenario of various test networks using different ACOPF formulations.

\begin{tabular}{|c|c|c|c|c|c|c|c|}
\hline \hline Network & BPFPV & BPFRV & BCFRV & BCFW & NIPAPV & NIRAPV & NIPARV \\
\hline \hline Network_01R-040 & 45 & 49 & 62 & 50 & 46 & 41 & 46 \\
\hline Network_03R-050 & 22 & 23 & 28 & 34 & 21 & 19 & 28 \\
\hline Network_05R-018 & 31 & 36 & 709 & 120 & 40 & 41 & 56 \\
\hline Network_07R-122 & 58 & 86 & 253 & 112 & 47 & 43 & 101 \\
\hline Network_09R-070 & 32 & 38 & 41 & 46 & 30 & 28 & 43 \\
\hline Network_12R-030 & 132 & 104 & 147 & 131 & 68 & 71 & 198 \\
\hline Network_81R-050 & 68 & 59 & 82 & 76 & 60 & 56 & 73 \\
\hline Network_84R-100 & 142 & 130 & 152 & 133 & 62 & 62 & 77 \\
\hline \hline
\end{tabular}

subproblem in BPFPV formulation has higher complexity than the subproblem in BCFRV and NIPARV formulations.

\section{CONCLUSION}

The paper presents seven different ACOPF formulations, each representing a unique optimization structure and sparsity. The work includes an explicit investigation of the impact of these ACOPF formulations on the performance of ACOPF algorithms. The paper employs the Interior-Point Line Search Algorithm for Nonlinear Programming implemented by IPOPT solver to evaluate these formulations. Results show that the choice of formulation, which is often overlooked, can significantly impact the computation time of an algorithm, especially for large-scale networks. Numerical results in the paper suggest a consistently superior performance by Nodal Injection Polar Admittance Polar Voltage (NIPAPV) and Nodal Injection Rectangular Admittance Polar Voltage (NIRAPV) formulations among different ACOPF formulations studied in this paper and across all ranges of test networks. The main contributing factors in the superior performance of NIPAPV and NIRAPV are the least sparsity of their optimization structures and modeling voltages in polar form. The complexity of the sub-problem of these two formulations compared to the other formulations has also consistently remained the least. 


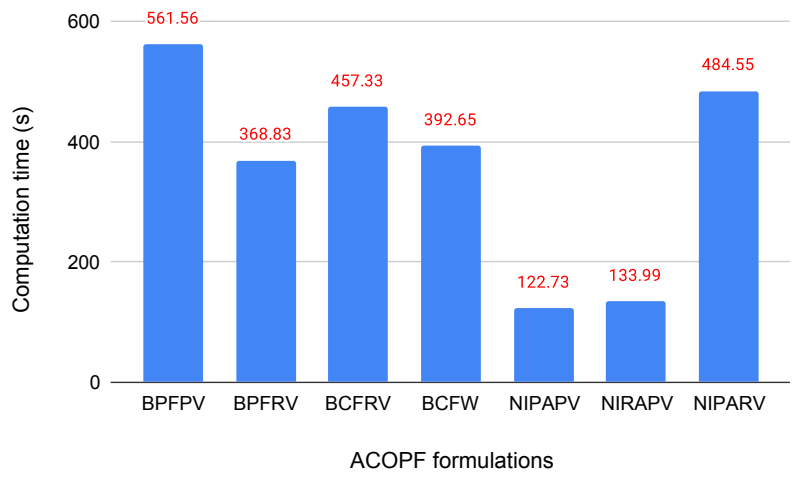

Fig. 4: The bar chart shows the computation time of IPLS algorithm to solve each formulation using the same scenario of Network_12R-030.

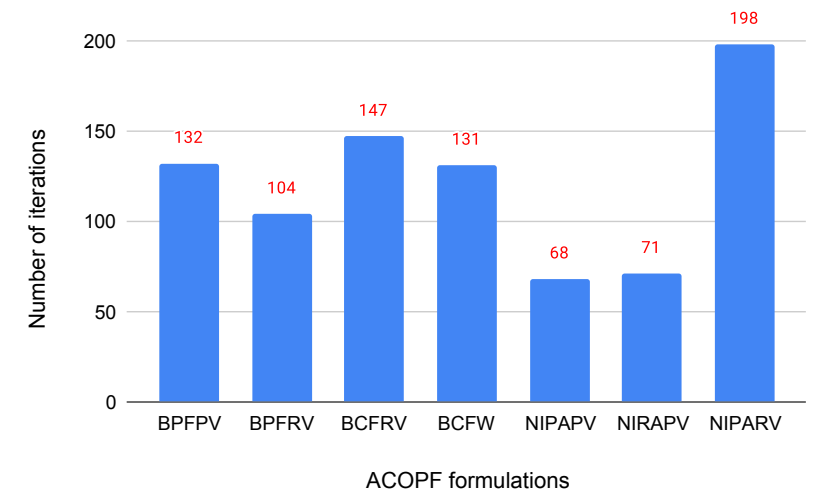

Fig. 5: The bar chart shows the number of iterations needed by IPLS Algorithm to solve each formulation for the same scenario of Network_12R-030.

\section{REFERENCES}

[1] M. B. Cain, R. P. O'neill, A. Castillo et al., "History of optimal power flow and formulations," Federal Energy Regulatory Commission, vol. 1, pp. 1-36, 2012.

[2] S. A. Sadat, D. Haralson, and M. Sahraei-Ardakani, "Security versus computation time in iv-acopf with socp initialization," in 2018 IEEE International Conference on Probabilistic Methods Applied to Power Systems (PMAPS), 2018, pp. 1-6.

[3] A. Castillo and R. P. O'Neill, "Computational performance of solution techniques applied to the acopf," Federal Energy Regulatory Commission, Optimal Power Flow Paper, vol. 5, 2013.

[4] Energy Information Administration, "Revenue from sales of electricity to ultimate customers," 2019. [Online]. Available: https://www.eia.gov/ electricity/annual/html/epa_02_03.html

[5] B. Stott, J. Jardim, and O. Alsaç, "Dc power flow revisited," IEEE Transactions on Power Systems, vol. 24, no. 3, pp. 1290-1300, 2009.

[6] S. A. Sadat and L. Fan, "Mixed integer linear programming formulation for chance constrained mathematical programs with equilibrium constraints," in 2017 IEEE Power Energy Society General Meeting, 2017, pp. $1-5$.

[7] S. A. Sadat, "Optimal bidding strategy for a strategic power producer using mixed integer programming," 2017.

[8] A. Castillo, P. Lipka, J.-P. Watson, S. S. Oren, and R. P. O’Neill, "A successive linear programming approach to solving the iv-acopf," IEEE Transactions on Power Systems, vol. 31, no. 4, pp. 2752-2763, 2015.

[9] S. A. Sadat and M. Sahraei-Ardakani, "Cascading failure mitigation via transmission switching," preprint, 2021.
[10] J. Lavaei and S. H. Low, "Zero duality gap in optimal power flow problem," IEEE Transactions on Power Systems, vol. 27, no. 1, pp. 92-107, 2012.

[11] R. Madani, S. Sojoudi, and J. Lavaei, "Convex relaxation for optimal power flow problem: Mesh networks," IEEE Transactions on Power Systems, vol. 30, no. 1, pp. 199-211, 2015.

[12] S. H. Low, "Convex relaxation of optimal power flow-part i: Formulations and equivalence," IEEE Transactions on Control of Network Systems, vol. 1, no. 1, pp. 15-27, 2014.

[13] H. Hijazi, C. Coffrin, and P. Van Hentenryck, "Convex quadratic relaxations for mixed-integer nonlinear programs in power systems," Mathematical Programming Computation, vol. 9, no. 3, pp. 321-367, 2017.

[14] R. P. O'Neill, A. Castillo, and M. B. Cain, "The IV formulation and linear approximations of the ac optimal power flow problem," FERC, 2012.

[15] R. Byrd, N. Gould, J. Nocedal, and R. Waltz, "An algorithm for nonlinear optimization using linear programming and equality constrained subproblems," Mathematical Programming, vol. 100, no. 1, pp. 27-48, 2004, cited By 70.

[16] S. A. Sadat, D. Haralson, and M. Sahraei-Ardakani, "Evaluation of various techniques to warm-start a successive linear programming algorithm for solving the iv acopf," in 2018 IEEE Power Energy Society General Meeting (PESGM), 2018, pp. 1-5.

[17] L. Sampath, B. V. Patil, H. Gooi, J. Maciejowski, and K. Ling, "A trust-region based sequential linear programming approach for ac optimal power flow problems," Electric Power Systems Research, vol. 165, pp. $134-143,2018$. [Online]. Available: http://www.sciencedirect. com/science/article/pii/S0378779618302955

[18] S. A. Sadat and M. Sahraei-Ardakani, "Initializing Successive Linear Programming Solver for ACOPF using Machine Learning," in 2020 IEEE North American Power Symposium (NAPS), 2020, pp. 1-6.

[19] Z. Yang, H. Zhong, Q. Xia, A. Bose, and C. Kang, "Optimal power flow based on successive linear approximation of power flow equations," IET Generation, Transmission Distribution, vol. 10, no. 14, pp. 3654-3662, 2016.

[20] P. Fortenbacher and T. Demiray, "Linear/quadratic programmingbased optimal power flow using linear power flow and absolute loss approximations," International Journal of Electrical Power \& Energy Systems, vol. 107, pp. 680 - 689, 2019. [Online]. Available: http://www.sciencedirect.com/science/article/pii/S0142061518325377

[21] Z. Yang, A. Bose, H. Zhong, N. Zhang, Q. Xia, and C. Kang, "Optimal reactive power dispatch with accurately modeled discrete control devices: A successive linear approximation approach," IEEE Transactions on Power Systems, vol. 32, no. 3, pp. 2435-2444, 2017.

[22] S. Mhanna and P. Mancarella, "An exact sequential linear programming algorithm for the optimal power flow problem," IEEE Transactions on Power Systems, pp. 1-1, 2021.

[23] D. K. Molzahn and I. A. Hiskens, A Survey of Relaxations and Approximations of the Power Flow Equations, 2019, vol. 4, no. 1-2. [Online]. Available: http://dx.doi.org/10.1561/3100000012

[24] B. Park, L. Tang, M. C. Ferris, and C. L. DeMarco, "Examination of three different acopf formulations with generator capability curves," IEEE Transactions on Power Systems, vol. 32, no. 4, pp. 2913-2923, 2017.

[25] J. Nocedal and S. Wright, "Numerical optimization. 2nd edn springer," New York, 2006.

[26] J. Kardos, D. Kourounis, O. Schenk, and R. Zimmerman, "Complete results for a numerical evaluation of interior point solvers for large-scale optimal power flow problems," 2020.

[27] M. Lu, "Optimization Methods in Electric Power Systems: Global Solutions for Optimal Power Flow and Algorithms for Resilient Design under Geomagnetic Disturbances," 2018.

[28] A. Wahid, S. A. Sadat, M. Ardakani, and A. Tajalli, "Power system emulator based on pll architecture," in 2020 IEEE International Symposium on Circuits and Systems (ISCAS), 2020, pp. 1-4.

[29] The U.S. Department of Energy, "Grid Optimization (GO) competition - Challenge I," 2019. [Online]. Available: https://gocompetition.energy. gov/challenges/challenge- 1

[30] K. Kim, C. G. Petra, and V. M. Zavala, "An asynchronous bundletrust-region method for dual decomposition of stochastic mixed-integer programming," SIAM Journal on Optimization, vol. 29, no. 1, 12019. [Online]. Available: https://www.osti.gov/biblio/1497321 